\title{
ON OUTPUT FEEDBACK NONLINEAR MODEL PREDICTIVE CONTROL USING HIGH GAIN OBSERVERS FOR A CLASS OF SYSTEMS
}

\author{
Lars Imsland ${ }^{*, 1}$ Rolf Findeisen ${ }^{* *}$ Eric Bullinger ${ }^{* *}$ \\ Frank Allgöwer ${ }^{* *, 2}$ Bjarne A. Foss * \\ * Department of Engineering Cybernetics, Norwegian University \\ of Science and Technology, 7491 Trondheim, Norway, \\ \{Lars.Imsland,Bjarne.Foss\}@itk.ntnu.no \\ ** Institute of Systems Theory in Engineering, University of \\ Stuttgart, 70550 Stuttgart, Germany, \\ \{findeise,bullinger,allgower\}@ist.uni-stuttgart.de
}

\begin{abstract}
In recent years, nonlinear model predictive control schemes have been derived that guarantee stability of the closed loop under the assumption of full state information. However, only limited advances have been made with respect to output feedback in connection to nonlinear predictive control. Most of the existing approaches for output feedback nonlinear model predictive control do only guarantee local stability. Here we consider the combination of stabilizing instantaneous NMPC schemes with high gain observers. For a special MIMO system class we show that the closed loop is asymptotically stable, and that the output feedback NMPC scheme recovers the performance of the state feedback in the sense that the region of attraction and the trajectories of the state feedback scheme are recovered for a high gain observer with large enough gain and thus leading to semi-global/non-local results.
\end{abstract}

Keywords: Output Feedback Stabilization, Nonlinear Model Predictive Control, High Gain Observers

\section{INTRODUCTION}

Model predictive control for systems described by nonlinear ODEs (NMPC) is an area that has received considerable attention over the past years. Several schemes guaranteeing stability under state feedback exist, see for example (Mayne et al., 2000; Allgöwer et al., 1999; De Nicolao et al., 2000) for recent reviews.

Fewer results are available in the case when not all states are directly measured. A common approach is to design a state feedback NMPC and to use a state observer to obtain an estimate of the system states used in the NMPC. In this case, little can

1 This work was done while LI visited the IST

2 corresponding author be said about the stability of the closed loop, since there exists no universal separation principle for nonlinear systems as it does for linear systems. In (Michalska and Mayne, 1995) a moving horizon observer is designed, that together with the NMPC scheme proposed in (Michalska and Mayne, 1993) is shown to lead to (semi-global) closed loop stability if no model-plant mismatch and disturbances are present. In (Magni et al., 1998), see also (Scokaert et al., 1997), local asymptotic stability for observer based discretetime nonlinear MPC is obtained for "weakly detectable" systems. However, these results are only of local nature, i.e. stability is guaranteed only for a sufficiently small observer error and no exact information about the required "level of smallness" 
is available.

In this article we propose the use of high gain observers in conjunction with instantaneous NMPC. For a special MIMO system class, the resulting control scheme does allow performance recovery of the state feedback controller if the observer gain is increased, i.e. the region of attraction and the rate of convergence of the output feedback scheme approach that of the state feedback scheme.

To show stability, we will mainly use results on separation principles for the considered class of systems as described in (Atassi and Khalil, 1999), see also (Teel and Praly, 1995; Esfandiari and Khalil, 1987). There, it is shown that a high-gain observer, in combination with a globally bounded state feedback can, under certain conditions such as locally Lipschitz continuity of the state feedback law, achieve recovery of asymptotic stability and performance.

The remainder of the paper is structured as follows. In Section 2 we specify the considered class of systems. Section 3 states the NMPC schemes considered for state feedback. In Section 4 the high-gain observer for state estimation is outlined. Section 5 contains the main results for the resulting closed loop system. These results are discussed in more detail in Section 6, and are illustrated with a simulation case study involving a mixedculture bio-reactor with competition and external inhibition in Section 7.

\section{SYSTEM CLASS}

We consider nonlinear systems of dimension $r+l$ of the form

$$
\begin{aligned}
\dot{x} & =A x+B \phi(x, z, u) \\
\dot{z} & =\psi(x, z, u) \\
y & =\left[\begin{array}{c}
C x \\
z
\end{array}\right]
\end{aligned}
$$

where $x(t) \in \mathbb{R}^{r}$ and $z(t) \in \mathbb{R}^{l}$ are the state variables and $y(t) \in \mathbb{R}^{p+l}$ are the measured outputs. Notice that we assume that all states $z$ are directly measured. The control input is assumed to be constrained, i.e. $u(t) \in \mathcal{U} \subset \mathbb{R}^{m}$ and $\mathcal{U}$ satisfies the following Assumption:

Assumption 1. $\mathcal{U} \subset \mathbb{R}^{m}$ is compact and the origin is contained in the interior of $\mathcal{U}$.

Hence $u$ is bounded and the equilibrium does satisfy the input constraint.

The $r \times r$ matrix $A, r \times p$ matrix $B$ and the $p \times r$ matrix $C$ have the following form:

$$
\begin{aligned}
A & =\text { blockdiag }\left[A_{1}, A_{2}, \ldots A_{p}\right] \\
A_{i} & =\left[\begin{array}{ccccc}
0 & 1 & 0 & \cdots & 0 \\
0 & 0 & 1 & \cdots & 0 \\
\vdots & & & & \vdots \\
0 & \cdots & \cdots & 0 & 1 \\
0 & \cdots & \cdots & \cdots & 0
\end{array}\right]_{r_{i} \times r_{i}}
\end{aligned}
$$

$$
\begin{aligned}
B & =\operatorname{blockdiag}\left[B_{1}, B_{2}, \ldots, B_{p}\right] \\
B_{i} & =\left[\begin{array}{llll}
0 & \cdots & 0 & 1
\end{array}\right]_{r_{i} \times 1}^{T} \\
C & =\operatorname{blockdiag}\left[C_{1}, C_{2}, \ldots, C_{p}\right] \\
C_{i} & =\left[\begin{array}{llll}
1 & 0 & \cdots & 0
\end{array}\right]_{1 \times r_{i}},
\end{aligned}
$$

i.e. the first part of the dynamics $x$ consists of $p$ integrator chains, with $r=r_{1}+\cdots+r_{p}$.

Assumption 2. The functions $\phi: \mathbb{R}^{r} \times \mathbb{R}^{l} \times \mathcal{U} \rightarrow$ $\mathbb{R}^{p}$ and $\psi: \mathbb{R}^{r} \times \mathbb{R}^{l} \times \mathcal{U} \rightarrow \mathbb{R}^{l}$ are assumed to be locally Lipschitz in their arguments over the domain of interest with $\phi(0,0,0)=0$ and $\psi(0,0,0)=0$. Additionally we assume, that $\phi$ is bounded in $x$ everywhere ${ }^{3}$.

One example of systems of this class are input affine nonlinear systems of the form

$$
\dot{\zeta}=f(\zeta)+g(\zeta) u, \quad y=h(\zeta)
$$

with full (vector) relative degree $\left(r_{1}, r_{2}, \ldots, r_{p}\right)$, that is, $\sum_{i=1}^{p} r_{i}=\operatorname{dim} \zeta$. Then, it is possible to find a coordinate transformation such that the system is described by (1a) and (1c), see (Isidori, 1985).

\section{NONLINEAR MPC STATE FEEDBACK}

The proposed output feedback controller consists of a suitable high gain observer to estimate the states and an instantaneous full state feedback NMPC controller. In the framework of predictive control, the input is defined by the solution of an open loop optimal control problem that has to be solved at all times. Here we assume that the input to the system is derived via the following NMPC open loop control problem:

\section{State feedback NMPC open loop optimal control problem: \\ Solve

$$
\min _{\bar{u}(\cdot)} J(x(t), z(t), \bar{u}(\cdot))
$$

subject to:

$$
\begin{aligned}
& \dot{\bar{x}}=A \bar{x}+B \phi(\bar{x}, \bar{z}, \bar{u}), \quad \bar{x}(0)=x(t) \\
& \dot{\bar{z}}=\psi(\bar{x}, \bar{z}, \bar{u}) \quad \bar{z}(0)=z(t) \\
& \bar{u}(\tau) \in \mathcal{U}, \quad \tau \in\left[0, T_{p}\right] \\
& \left(\bar{x}\left(T_{p}\right), \bar{z}\left(T_{p}\right)\right) \in \Omega
\end{aligned}
$$

with the cost functional:

$$
\begin{array}{r}
J(x(t), z(t), \bar{u}(\cdot)):=\int_{0}^{T_{p}} F(\bar{x}(\tau), \bar{z}(\tau), \bar{u}(\tau)) d \tau \\
+E\left(\bar{x}\left(T_{p}\right), \bar{z}\left(T_{p}\right)\right) .
\end{array}
$$

\footnotetext{
3 The assumption on global boundedness of $\phi$ in $x$ is strong. But note that global boundedness in most cases can be practically achieved by saturation outside a compact subspace of $\mathbb{R}^{r}$ of interest.
} 
The bar denotes internal controller variables and $\bar{x}(\cdot)$ is the solution of $(4 \mathrm{a})-(4 \mathrm{~b})$ driven by the input $\bar{u}(\cdot):\left[0, T_{p}\right] \rightarrow \mathcal{U}$ with initial condition $x(t), z(t)$ at time $t$. The stage cost to be minimized over the control horizon $T_{p}$ is given by $F(\bar{x}, \bar{z}, \bar{u})$. We assume, that $F$ satisfies:

Assumption 3. $\quad F: \mathbb{R}^{r} \times \mathbb{R}^{l} \times \mathcal{U} \rightarrow \mathbb{R}$ is continuous in all arguments with $F(0,0,0)=0$ and $F(x, z, u)>$ $c_{F}\|(x, z, u)\|_{2}^{2} \forall(x, z, u) \in \mathbb{R}^{r} \times \mathbb{R}^{l} \times \mathcal{U}, c_{F} \in \mathbb{R}^{+}$.

The constraint (4d) in the NMPC open loop optimal control problem forces the final predicted state at time $\tau=T_{P}$ to lay in a terminal region denoted by $\Omega$ and is thus often called terminal region constraint. In the cost functional $J$, the deviation from the origin of the final predicted state is penalized by the final state penalty term E.

Notice, that for simplicity we only consider input constraints (besides the terminal state constraint). An extension to more general cases is possible.

We denote the solution to the optimal control problem by $\bar{u}^{\star}(\cdot ; x(t), z(t))$. The system input used is given by the "first" value of the instantaneous solution of the open loop optimal control problem $\bar{u}^{\star}(\cdot ; x(t), z(t))$ :

$$
u(x(t), z(t)):=\bar{u}^{\star}(\tau=0 ; x(t), z(t)) .
$$

Several NMPC concepts that guarantee stability and that are similar to this setup have been proposed (Mayne and Michalska, 1990; Chen and Allgöwer, 1998; Jadbabaie et al., 1999a), see (Mayne et al., 2000) for a review. Similar results have also been obtained for the discrete time case (Meadows and Rawlings, 1993; De Nicolao et al., 1996; Findeisen, 1997).

Under the assumption that $T_{p}, E, F$ are suitably chosen (see for example (Chen and Allgöwer, 1998; Jadbabaie et al., 1999a; Mayne et al., 2000)) the origin of the nominal closed-loop system with the input (6) is asymptotically stable and the region of attraction $\mathcal{R} \subset \mathbb{R}^{r} \times \mathbb{R}^{l}$ contains the set of states for which the open loop optimal control problem has a solution.

We will assume that $T_{p}, E, F$ are chosen such, that the state feedback defined via the NMPC open loop optimal control problem and (6) is locally Lipschitz and stabilizes the system, i.e. we assume that the following assumption holds:

Assumption 4. The instantaneous state feedback defined by $u(x(t), z(t)):=\bar{u}^{\star}(\tau=0 ; x(t), z(t))$ is locally Lipschitz in $(x, z)$ and asymptotically stabilizes the system (1) with a region of attraction $\mathcal{R}$.

Remark 1. Notice that we assume an instantaneous state feedback, i.e. the solution of the open loop optimal control problem is available with no computational delay.

Remark 2. In general, the solution of an optimal control problem (and hence, the state feedback defined in Assumption 4) can be non-Lipschitz in the initial values. In (Jadbabaie et al., 1999b) it is noted that the control of an unconstrained NMPC problem is continuous given that the minimum value of the cost function is attained, and that some regularity conditions on $f, F$ and $E$, similar to those in this paper hold. Some results concerning the Lipschitz continuity of optimal control problems have been reported (Hager, 1979; Dontchev and Hager, 1993). However the resulting conditions are difficult to check and do only consider the Lipschitz continuity of the control law in terms of system parameters and not initial conditions.

\section{USED HIGH GAIN OBSERVER}

The proposed (partial state) observer is a high gain observer as in (Atassi and Khalil, 1999; Teel and Praly, 1995; Tornambè, 1992). It is of the following form:

$$
\begin{aligned}
\dot{\hat{x}} & =A \hat{x}+B \phi(\hat{x}, z, u)+H\left(y_{x}-C \hat{x}\right) \\
\text { where } H & =\operatorname{blockdiag}\left[H_{1}, \ldots, H_{p}\right] \\
H_{i}^{T} & =\left[\begin{array}{ll}
\alpha_{1}^{(i)} / \epsilon, & \left.\alpha_{2}^{(i)} / \epsilon^{2}, \ldots, \alpha_{n}^{(i)} / \epsilon^{r_{i}}\right]
\end{array}\right.
\end{aligned}
$$

and the $\alpha_{j}^{(i)}$ s are such, that the roots of

$$
s^{n}+\alpha_{1}^{(i)} s^{n-1}+\cdots+\alpha_{n-1}^{(i)} s+\alpha_{n}^{(i)}=0, i=1, \ldots, p
$$

are in the open left half plane. $y_{x}$ are the measurements of the $x$ states, $y_{x}=C x$. $\frac{1}{\epsilon}$ is the high-gain parameter, which can be seen as a time scaling for the observer dynamics (7). A, B, $C$ and $\phi$ are the same as in (1).

Remark 3. We only have to design an observer for the $x$ states of the integrator chain, since the $z$ states are assumed to be measured directly.

\section{OUTPUT FEEDBACK NMPC USING HIGH GAIN OBSERVERS}

Now we are ready to state our main result, which follows from (Atassi and Khalil, 1999) and in part from (Teel and Praly, 1995). In short, we can claim recovery of performance and asymptotic stability (see Remark 4) of the origin if the parameter $\epsilon$ in the observer is chosen small enough (that is, the observer is fast enough).

Consider the closed loop system given by (1a)-(1c) with the control given by the NMPC controller as described in Section 3 satisfying Assumption 4 
and using the observed state $\hat{x}$ from the observer (7) instead of $x$. Notice that the state feedback NMPC is assumed to asymptotically stabilize the system with a region of attraction $\mathcal{R}$ and that the value of the input is bounded, since the input constraint set $\mathcal{U}$ is compact.

Theorem 1. Let $\mathcal{S}$ be any compact set contained in the interior of $\mathcal{R}$. Then there exists a (small enough) $\epsilon^{*}>0$ such that for all $0<\epsilon \leq \epsilon^{*}$, the closed loop system is asymptotically stable with a region of attraction of at least $\mathcal{S}$. Further, the performance of the state feedback NMPC controller is recovered as $\epsilon$ decreases.

Proof 1. The asymptotic stability follows directly from the proofs of Theorem 1, 2 and 4 in (Atassi and Khalil, 1999) under the given assumptions.

Theorem 3 in (Atassi and Khalil, 1999) shows that the trajectories of the controlled system using the observed state in the controller, converge uniformly to the trajectories of the controlled system using the true state in the controller, as $\epsilon \rightarrow 0$. Hence, for $\epsilon$ small enough, the trajectories (and hence the performance) of the state feedback NMPC are recovered.

In the following we briefly specify what is meant by recovery of performance.

Remark 4. [Trajectory convergence/performance recovery] Define $\chi_{o}(t, \epsilon)$ as the state of the system at time $t$ using the output feedback NMPC controller with $\chi_{o}(0, \epsilon)=(x(0), z(0))$ as initial condition and using $\hat{x}$ from the observer (started with the initial condition $\hat{x}(0))$ instead of $x$ in the controller. Define $\chi_{s}(t)$ as the state of the system at time $t$ using the state feedback NMPC controller with $\chi_{s}(0, \epsilon)=(x(0), z(0))$ as initial condition. Then, for any $\xi>0$, there exists an $\tilde{\epsilon}^{*}$ such that for all $0<\epsilon \leq \tilde{\epsilon}^{*},\left\|\chi_{o}(t, \epsilon)-\chi_{s}(t)\right\| \leq \xi, \forall t>0$. This trajectory convergence in $\epsilon$ leads to what is meant by performance recovery and implies for example conformance to constraints, recovery of the region of attraction and rate of convergence.

If state constraints are included in the NMPC formulation, then we see that the state constraints are respected by the output feedback controller within any degree of accuracy by making $\epsilon$ small enough. However restrictive state constraints in the NMPC controller may lead to violation of the Lipschitz continuity condition on the controller, and hence are not included.

Remark 5. (Robustness). In the separation principle in (Atassi and Khalil, 1999), robustness to some modeling error in the observer (that is, in $\phi)$ is shown. In our case, it makes no sense to consider the observer model as incorrect, since we assume that we have perfect model knowledge in the NMPC controller. However, it indicates that the observer structure has some robustness properties (see (Teel and Praly, 1995, Lemma 2.4)).

\section{DISCUSSION}

The result in Section 5 is based on the assumption that the NMPC controller is time continuous/instantaneous. In a real application, it is of course not possible to solve the nonlinear optimization problem instantaneously. Instead, it typically will be solved only at some sampling instants. The first part of the computed control is then applied to the system, until the next sampling instant. Further, some time is needed to compute the optimal solution of the optimization problem, thus the computed control to some degree is based on old information, introducing delay in the closed loop.

In a transient phase, due to the peaking phenomenon, the observed state may be outside the region where the NMPC optimization problem has a feasible solution. In this case the input should be assigned some fallback value. The high gain observer structure and the results of (Atassi and Khalil, 1999) ensure that $\epsilon$ can be chosen small enough so that the observer state converges to the true state before the true state leaves the region of attraction (and hence the feasibility area) of the NMPC controller. This follows from the assumption of bounded controls (Esfandiari and Khalil, 1992).

\section{EXAMPLE}

To show recovery of performance we consider the control of a continuous mixed culture bioreactor as presented in (Hoo and Kantor, 1986). The model considered describes the dynamics of a culture of two cell strains, in the following called species 1 and 2, that have different sensitivity to an external growth-inhibiting agent. The interactions of the two cell populations is illustrated in Figure 1. The cell density of the inhibitor resistant

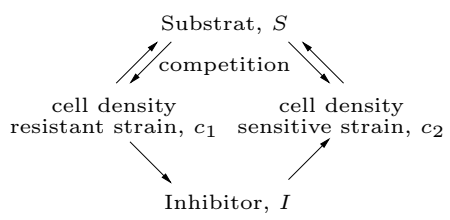

Fig. 1. Strain/inhibitor interactions.

strain is denoted by $c_{1}$, the cell density of the inhibitor sensitive strain is denoted with $c_{2}$ and the substrate and inhibitor concentrations in the reactor are denoted by $S$ and $I$. Following the description in (Hoo and Kantor, 1986) a reduced third order model of the following form can be obtained: 


$$
\begin{aligned}
\frac{d c_{1}}{d t} & =\mu_{1}(S) c_{1}-c_{1} u_{1}, \\
\frac{d c_{2}}{d t} & =\mu_{2}(S, I) c_{2}-c_{2} u_{1}, \\
\frac{d I}{d t} & =-p c_{1} I+u_{2}-I u_{1} .
\end{aligned}
$$

The inputs are the dilution rate $u_{1}=D$ and the inhibitor addition rate $u_{2}=D I_{f}$, with $I_{f}$ being the inhibitor inlet concentration. The deactivation constant of the inhibitor for species 2 is denoted by $p . \mu_{1}(S)$ and $\mu_{2}(S, I)$ are the specific growth rates given by:

$$
\mu_{1}(S)=\frac{\mu_{1, M} S}{K+S}, \quad \mu_{2}(S, I)=\frac{\mu_{2, M} S}{K+S} \frac{K_{I}}{K_{I}+I} .
$$

where $K, K_{I}, \mu_{1, M}$ and $\mu_{2, M}$ are constant parameters. The substrate concentration is given by:

$$
S=S_{f}-\frac{c_{1}}{Y_{1}}-\frac{c_{2}}{Y_{2}} .
$$

Here $Y_{1}, Y_{2}$ are the yields of the species and $S_{f}$ is the substrate inlet concentration. The control objective is to stabilize the steady state given by $c_{1 s}=0.016 \mathrm{~g} / \mathrm{l}, c_{2 s}=0.06 \mathrm{~g} / \mathrm{l}, I_{s}=0.005 \mathrm{~g} / \mathrm{l}$. We will use the output-feedback NMPC scheme as outlined in the previous sections to achieve this. If we use

$$
y^{T}=\left[\ln \frac{c_{1}}{c_{2}}, c_{1}\right]
$$

as measured outputs and if we make the following coordinate transformation

$$
x_{1}=\ln \frac{c_{1}}{c_{2}}, \quad x_{2}=\mu_{1}(S)-\mu_{2}(S, I), \quad z=c_{1}
$$

the transformed system is of the model class assumed in Section 2:

$$
\begin{aligned}
\dot{x}_{1} & =x_{2}, \\
\dot{x}_{2} & =\phi\left(x_{1}, x_{2}, z, u_{1}, u_{2}\right), \\
\dot{z} & =\psi\left(x_{1}, x_{2}, z, u_{1}, u_{2}\right) .
\end{aligned}
$$

As NMPC scheme we use the so called quasiinfinite horizon NMPC strategy as presented in (Chen and Allgöwer, 1998) with the sampling time set to zero. As cost $F$ we use the quadratic deviation of the states and the inputs in the new coordinates from their steady state values. For simplicity unit weights on all states and inputs are considered. The horizon $T_{p}$ was set to $20 \mathrm{hr}$. A quadratic upper bound $E$ on the infinite horizon cost and a terminal region $\Omega$ that satisfy the assumptions of (Chen and Allgöwer, 1998) and with this the assumptions made in Section 3 are calculated using LMI/PLDI-techniques (Boyd et al., 1994). The piecewise linear differential inclusion (PLDI) representing the dynamics in a neighborhood of the origin was found using the methods described in (Slupphaug et al., 2000).

The states $x_{1}$ and $x_{2}$ are estimated from the measurements of $x_{1}$ and $z$ via a high gain observer as described in Section 4. The parameters $\alpha_{1}$ and $\alpha_{2}$ are chosen to $\alpha_{1}=\sqrt{2}, \alpha_{2}=1$. To show the recovery of performance we consider different values of the high-gain parameter $\epsilon$ of the observer. Notice, that in real applications the value of $\epsilon$ should not be decreased to much, since there is always a trade off between the performance recovery and the robustness to noise and modeling errors. For all cases the observer is started with the correct values for $x_{1}$ and $x_{3}$ (since they can be directly obtained from the measurements), whereas $x_{2}$ is assumed unknown and initialized with the steady state value. Figure 2 shows closed-loop system trajectories for different observer gains $\frac{1}{\epsilon}$ in comparison to a state feedback NMPC controller (using no high gain observer for state recovery). As can be

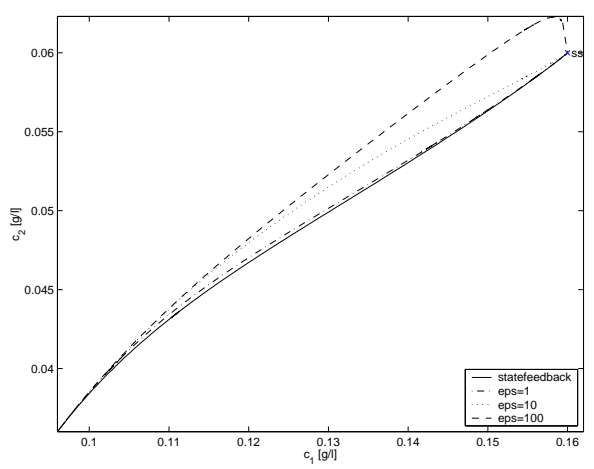

Fig. 2. Phase plot of $c_{1}$ and $c_{2}$ (SS... steady state)

seen, the higher the observer gain gets, the more performance is recovered by the output feedback controller, i.e. the trajectories tend towards the "optimal" state feedback ones. Figure 3 exemplifies the time behavior of the inhibitor concentration $I$ (related to the unmeasured state $x_{2}$ ) and the inhibitor addition rate $D I_{F}$ (input $u_{2}$ ) for different values of $\epsilon$. Additionally, we plot the real
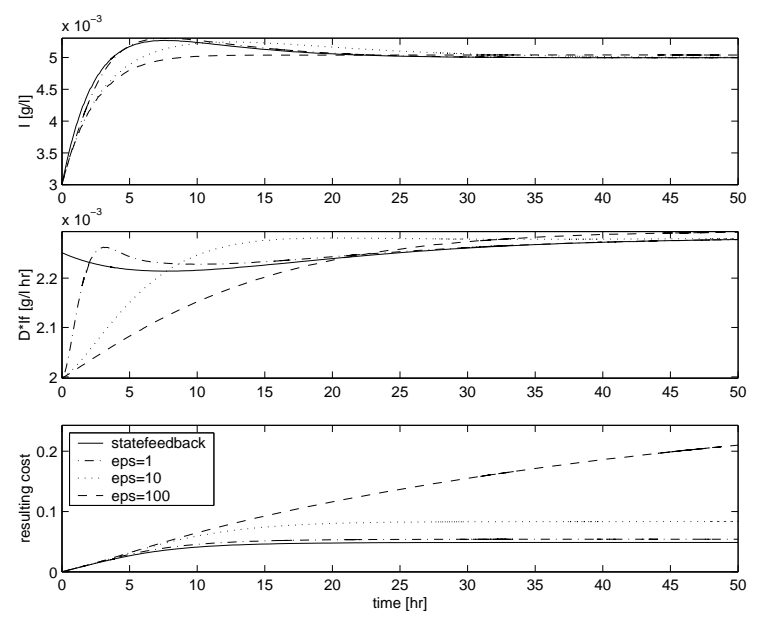

Fig. 3. Trajectories of $I, u_{1}$ and summed up cost

cost that occurs, i.e. the integrated quadratic error between the steady state values for the states and inputs in transformed coordinates. As one can see, the state feedback controller leads to the lowest 
cost. The cost of the output feedback controller approaches the cost of the state feedback controller for decreasing $\epsilon$, which shows the recovery of performance.

Notice, that we use relatively low gains of the observer, meaning that $\epsilon$ is large. Higher observer gains can lead to problems in case of measurement noise. This is one of the main limitations of using high gain observers for state estimation. We do not go into details here. The given example underpins the derived results on performance recovery for the high-gain observer based NMPC strategy.

\section{CONCLUSIONS}

Nonlinear model predictive control has received considerable attention during the recent years. However no significant progress with respect to the output feedback case has been made. The existing solutions are either of local nature (Scokaert et al., 1997; Magni et al., 1998) or difficult to implement (Michalska and Mayne, 1995). In this paper we have outlined, that using results from (Atassi and Khalil, 1999), "semi-global" stability results on a bounded region of attraction and recovery of performance can be achieved utilizing NMPC and a suited high gain observer for a class of systems. The price to pay is that one has to assume that the NMPC control law is local Lipschitz in the states over the domain of interest and that only systems with "directly measurable internal dynamics" can be considered. However, in practical problems the locally Lipschitz assumption is often not a significant problem. Additionally, one should notice the inherent problem of high gain observers with respect to measurement noise. The given result must be seen as a first step towards the development of a practical relevant output feedback NMPC scheme.

\section{REFERENCES}

Allgöwer, F., T.A. Badgwell, J.S. Qin, J.B. Rawlings and S.J. Wright (1999). Nonlinear predictive control and moving horizon estimation - An introductory overview. In: Advances in Control, Highlights of ECC'99 (P. M. Frank, Ed.). pp. 391-449. Springer.

Atassi, A.N. and H. Khalil (1999). A separation principle for the stabilization of a class of nonlinear systems. IEEE Trans. Automat. Contr. 44(9), 1672-1687.

Boyd, S., L. El Ghaoui, E. Feron and V. Balakishnan (1994). Linear Matrix Inequalities in System and Control Theory. SIAM. Philadelphia.

Chen, H. and F. Allgöwer (1998). Nonlinear model predictive control schemes with guaranteed stability. In: Nonlinear Model Based Process Control (R. Berber and C. Kravaris, Eds.). Kluwer Academic Publishers, Dodrecht. pp. 465-494.

De Nicolao, G., L. Magni and R. Scattolini (1996). Stabilizing nonlinear receding horizon control via a nonquadratic terminal state penalty. In: Symposium on Control, Optimization and Supervision, CESA'96 IMACS Multiconference. Lille. pp. 185-187.
De Nicolao, G., L. Magni and R. Scattolini (2000). Stability and robustness of nonlinear receding horizon control. In: Nonlinear Predictive Control (F. Allgöwer and A. Zheng, Eds.). Birkhäuser. pp. 3-23.

Dontchev, A. and W. Hager (1993). Lipschitzian stability in nonlinear control and optimization. SIAM J. Control Optim. 31(3), 569-603.

Esfandiari, F. and H.. Khalil (1987). Observer-based design of uncertain systems: recovering state feedback robustness under matching conditions. In: Proc. of the 25th Anual Allerton Conf. on Comunication, Control, and Computing. Monticello, IL. pp. 97-106.

Esfandiari, F. and H. Khalil (1992). Output feedback stabilization of fully linearizable systems. Int. J. Contr. 56(5), 1007-1037.

Findeisen, R. (1997). Suboptimal nonlinear model predictive control. Master's thesis. University of WisconsinMadison.

Hager, W.E. (1979). Lipschitz continuity for constrained processes. SIAM J. Contr. Optim. 17(3), 321-338.

Hoo, K. A. and J. C. Kantor (1986). Global linearization and control of a mixed culture bioreactor with competition and external inhibition. Math. Biosci. 82, 4362 .

Isidori, A. (1985). Nonlinear Control Systems: An Introduction. Springer-Verlag. Berlin.

Jadbabaie, A., J. Yu and J. Hauser (1999a). Stabilizing receding horizon control of nonlinear systems: A control Lyapunov approach. In: Proc. Amer. Contr. Conf.. ACC. San Diego. pp. 1535-1539.

Jadbabaie, A., J. Yu and J. Hauser (1999b). Unconstrained receding horizon control of nonlinear systems. In: Proc. 38th IEEE Conf. Decision Contr.. San Diego. pp. 3376-3381.

Magni, L., D. De Nicolao and R. Scattolini (1998). Output feedback receding-horizon control of discrete-time nonlinear systems. In: Preprints of the 4 th Nonlinear Control Systems Design Symposium 1998 - NOLCOS'98. IFAC. pp. 422-427.

Mayne, D.Q. and H. Michalska (1990). Receding horizon control of nonlinear systems. IEEE Trans. Automat. Contr. 35(7), 814-824.

Mayne, D.Q., J.B. Rawlings, C.V. Rao and P.O.M. Scokaert (2000). Constrained model predictive control: stability and optimality. Automatica 26(6), 789814.

Meadows, E.S. and J.B. Rawlings (1993). Receding horizon control with an infinite horizon. In: Proc. Amer. Contr. Conf.. San Francisco. pp. 2926-2930.

Michalska, H. and D.Q. Mayne (1993). Robust receding horizon control of constrained nonlinear systems. IEEE Trans. Automat. Contr. AC-38(11), 16231633.

Michalska, H. and D.Q. Mayne (1995). Moving horizon observers and observer-based control. IEEE Trans. Automat. Contr. 40(6), 995-1006.

Scokaert, P.O.M., J.B. Rawlings and E.S. Meadows (1997). Discrete-time stability with perturbations: Application to model predictive control. Automatica 33(3), 463-470.

Slupphaug, O., L. Imsland and B. Foss (2000). Uncertainty modelling and robust output feedback control of nonlinear discrete systems: a mathematical programming approach. International Journal of Robust and Nonlinear Control 10(13), 1129-1152.

Teel, A. and L. Praly (1995). Tools for semiglobal stabilization by partial state and output feedback. SIAM J. Contr. Optim. 33(5), 1443-1488.

Tornambè, A. (1992). High-gain observers for non-linear systems. Int. J. Sys. Sci. 23(9), 1475-1489. 